\title{
Novel Contamination and Gas Sensor Materials from Amphiphilic Polymer-Grafted Carbon Black
}

\author{
Haruo Morohashi, ${ }^{1}$ Taro NAKANOYA, ${ }^{1}$ Hiromitsu IwATA, ${ }^{1}$ \\ Takeshi YAMAUCHI, ${ }^{1}$ and Norio TSUBOKAWA ${ }^{2,3, \dagger}$ \\ ${ }^{1}$ Graduate School of Science and Technology, Niigata University, \\ 8050, Ikarashi 2-nocho, Niigata 950-2181, Japan \\ ${ }^{2}$ Department of Material Science and Technology, Faculty of Engineering, Niigata University, \\ 8050, Ikarashi 2-nocho, Niigata 950-2181, Japan \\ ${ }^{3}$ Center for Transdisciplinary Research, Niigata University, 8050, Ikarashi 2-nocho, Niigata 950-2181, Japan
}

(Received October 12, 2005; Accepted January 26, 2006; Published May 17, 2006)

\begin{abstract}
The responses of electric resistance of composites prepared from amphiphilic polymer-grafted carbon blacks to contamination in solution and solvent vapor were investigated. The electric resistance of the composite prepared from poly( $N$-isopropylacrylamide) (PNIPAM)-grafted carbon black drastically increased when the composite was dipped in $n$-hexane containing contamination, such as methanol, THF, dioctyl phthalate, and chloroform, and returned to the initial resistance when it was transferred to dry air. The logarithm of the electric resistance of the composite was linearly proportional to chloroform concentration in $n$-hexane. The electric resistance of the composite drastically increased in organic polar solvent vapor, such as methanol, THF, and chloroform and returned to the initial resistance when it was transferred to dry air. But the response to non-polar solvent vapor, such as $n$-hexane, was very small. The logarithm of electric resistance of the composite was linearly proportional to humidity. In addition, electric resistance of the composite prepared from poly(diethylacrylamide) (PDEAA)-grafted carbon black also drastically increased when the composite was dipped in $n$-hexane containing contamination, such as methanol, trichloroethane, THF, chloroform, and benzene, and returned to the initial resistance when it was transferred to dry air. Based on the results, it was found that the composites could be used as a novel contamination sensor in $n$-hexane and gas sensor. [doi:10.1295/polymj.PJ2005157]

KEY WORDS Carbon Black / Surface Grafting of Polymer / Conductive Composite / Amphiphilic Polymer / Contamination Sensor / Gas Sensor /
\end{abstract}

It has been reported that the grafting of various vinyl polymers onto a carbon black surface is successfully achieved by the polymerization of vinyl monomers initiated by initiating groups previously introduced onto the surface. ${ }^{1-3}$ The polymer-grafted carbon blacks thus obtained were easily and uniformly dispersed in polymer matrices. ${ }^{1-3}$ It is well known that a composite prepared from vinyl polymer-grafted carbon black, which are crosslinked with a variety of crosslinking agents, show a large positive temperature coefficient of electric resistance, i.e., PTC, near the glass transition temperature of the matrix polymer.

Recently, we have reported that the electric resistance of the composite from crystalline polymer-grafted carbon black drastically increased at the melting point of the grafted polymer. ${ }^{4}$ It is considered that this is due to a widening of the gaps between the carbon black particles based on the melting of grafted and matrix polymer.

We have also reported that the electric resistance of composite from polymer-grafted carbon black drastically increased in organic solvent vapor, which are good solvents of the grafted chain, but not in poor sol- vent vapors, and returned to the initial resistance when it was transferred to dry air. ${ }^{5-8}$ On the other hand, Narkis et $a l .{ }^{9}$ have reported the solvent sensing by use of conductive carbon black/polymer composite. Lews et al. ${ }^{10,11}$ and Zhang et al. ${ }^{12-15}$ have also reported the solvent vapor sensing behavior of carbon black/amorphous polymer composites.

In addition, we have pointed out that the conductive composite prepared from poly( $\varepsilon$-caprolactone)grafted, poly(ethylene glycol)-grafted, poly(ethylene adipate)-grafted and polyamide-block-poly(ethylene oxide)-grafted carbon black can be used as a contamination sensor in $n$-hexane. ${ }^{16}$

These phenomena may be due to the swelling based on the absorption and adsorption of solvent by matrix polymer. That is, the increase of the electric resistance in solvent vapors is considered that the matrix polymer is swelled when the polymer absorbed a contamination, and it may cut conducting circuit of the carbon black.

In this study, based on the above considerations, the electric resistance response of composites prepared from amphiphilic polymer, such as poly $(N$-isopropyl-

${ }^{\dagger}$ To whom correspondence should be addressed (Tel/Fax: +81-25-262-6779, E-mail: ntsuboka@eng.niigata-u.ac.jp). 
acrylamide) (PNIPAM) and poly(diethylacrylamide) (PDEAA), and these amphiphilic polymer-grafted carbon blacks to various contaminations in $n$-hexane and solvent vapors was investigated. In addition, the relationship between the electric resistance of composite and contamination in $n$-hexane or relative humidity was also discussed.

\section{EXPERIMENTAL}

\section{Carbon Black}

The conductive carbon black used was Porousblack (BET surface area, $447 \mathrm{~m}^{2} / \mathrm{g}$; particle size, $41 \mathrm{~nm}$ ) obtained from Asahi Carbon Co., Ltd., Japan. The content of phenolic hydroxyl and carboxyl groups on the surface was $0.30 \mathrm{mmol} / \mathrm{g}$ and $0 \mathrm{mmol} / \mathrm{g}$, respectively. The carbon black was dried in vacuo at $110^{\circ} \mathrm{C}$ for $48 \mathrm{~h}$ before use.

\section{Materials and Reagents}

$N$-Isopropylacrylamide (NIPAM) and 2,2'-azobisisobutyronitrile (AIBN) obtained from Wako Pure Chemical Co., Ltd. were recrystallized and dried in vacuo at room temperature. $N, N$-Diethylacrylamide (DEAA) was distilled under reduced pressure. Tetrahydrofuran (THF), $n$-hexane and other solvents were purified using standard methods.

\section{Grafting of Amphiphilic Polymers onto Carbon Black} Surface by Radical Trapping

The grafting of polymers, such as PNIPAM and PDEAA onto carbon black surface were achieved through the trapping of PNIPAM and PDEAA growing radicals formed during the polymerization initiated by AIBN in the presence of carbon black as shown in Scheme 1. Into a $100 \mathrm{~mL}$ flask, $1.0 \mathrm{~g}$ of carbon black, $4.0 \mathrm{~g}$ of NIPAM (or DEAA), $0.02 \mathrm{~g}$ of AIBN and $10 \mathrm{~mL}$ THF were charged and the reaction mixture was stirred with a magnetic stirrer under nitrogen at $60^{\circ} \mathrm{C}$ for $6 \mathrm{~h}$. In order to remove ungrafted polymer and AIBN from the product, the mixture was centrifuged and supernatant solution was removed. Then the precipitated carbon black was extracted with THF by use of a Soxhlet apparatus.
The amount of polymer grafted onto the carbon black surface was determined by weight loss when polymer-grafted carbon black was heated under nitrogen at a scan rate of $10^{\circ} \mathrm{C} / \mathrm{min}$ from 25 to $500^{\circ} \mathrm{C}$ by use of a thermal analyzer. The percentage of grafting was determined by the following equation:

$$
\text { Grafting }(\%)=(A / B) \times 100,
$$

where $A$ is PNIPAM (or DEAA) grafted (g) and $B$ is carbon black charged $(\mathrm{g})$.

\section{Preparation of Sensor Materials}

The preparation of composite resistor from polymer-grafted carbon black was carried out as follows. Into a test tube, $0.025 \mathrm{~g}$ of polymer-grafted carbon black was dispersed in a small amount of THF and $0.10 \mathrm{~g}$ of polymer was added. Then, the dispersion was irradiated under ultrasonic wave for $10 \mathrm{~min}$ and it was stirred at room temperature using a magnetic stirrer to produce a paste of composite (the content of carbon black was $20 \mathrm{wt} \%$ ). The resulting paste of composite was coated onto a comb-like electrode, which has an area of $8 \times 10 \mathrm{~mm}$, prepared by the screen printing of conductive $\mathrm{Ag} / \mathrm{Pd}$ paste onto a ceramic alumina plate. The comb-like electrode used in this study was shown in Figure 1. The comb-like electrode, which applied a paste of composite, was dried in air at room temperature for $1 \mathrm{~h}$ and then dried in vacuo at room temperature for $24 \mathrm{~h}$. The coated composite has an area of $5 \times 7 \mathrm{~mm}$. The thickness of the composite was about $10 \mu \mathrm{m}^{5-8}$

\section{Measurement of Electric Resistance}

The electric resistance of the composite in $n$-hexane was measured by dipping the comb-like electrode coated with composite in $n$-hexane solutions containing a contamination. ${ }^{9}$

On the other hand, the electric resistance of the composite in solvent vapor was measured by hanging the comb-like electrode in a glass tube containing pure solvent at the bottom. The direct current electric resistance of electrode coated with composite was measured as a function of time. Resistance measurements were performed using a simple two-point configura-

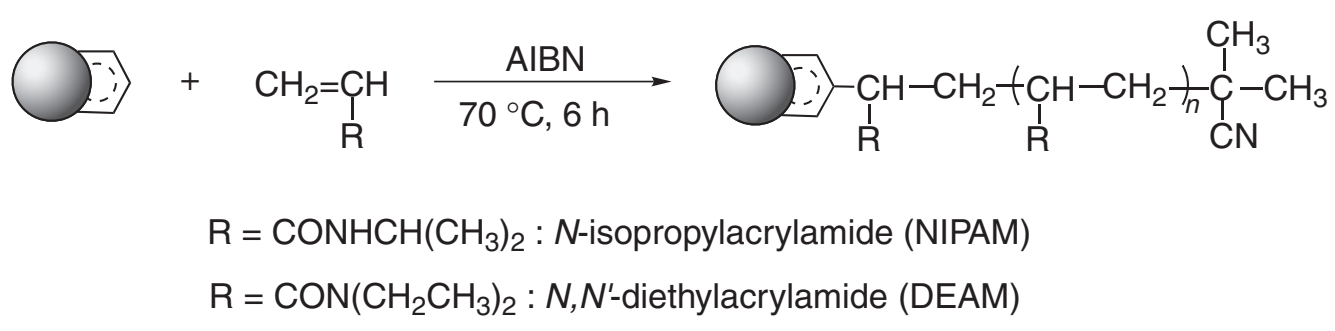

Scheme 1. 


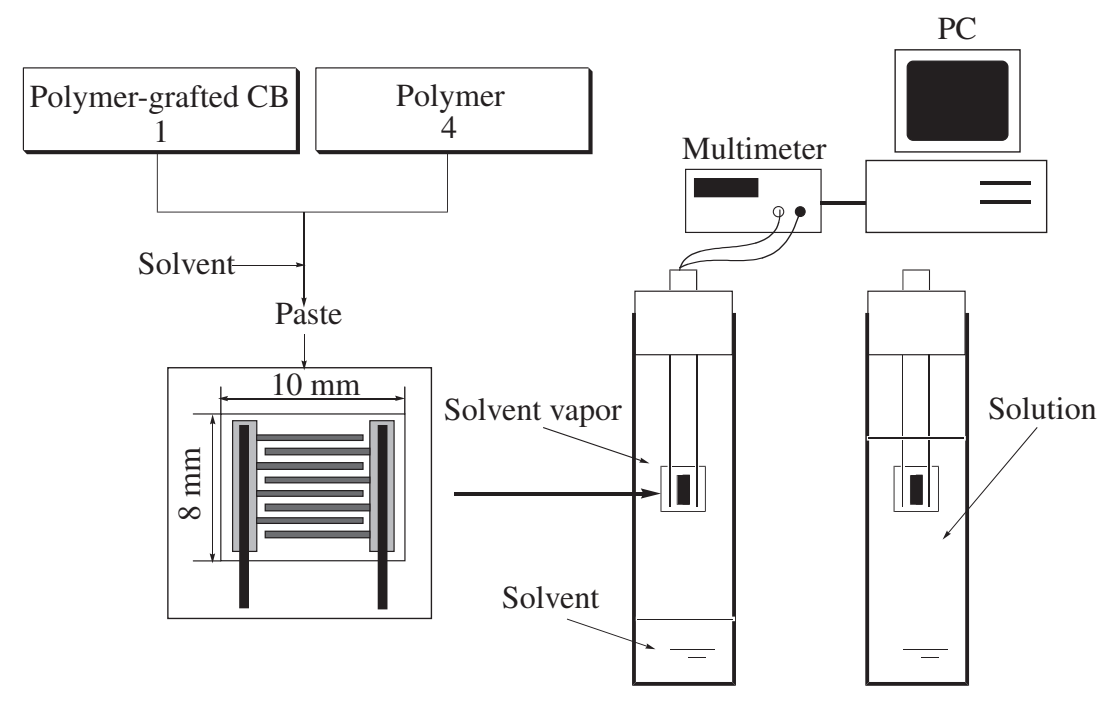

Figure 1. Comb-like electrode and apparatus for the measurement of electric resistance of composite in solution and solvent vapor.

tion. The electrode was connected directly to a digital multimeter (made by Advantest Co. Ltd., Japan; type R6871E-DC) via a ribbon cable, and the resistance was read by a personal computer.

\section{RESULTS AND DISCUSSION}

Grafting of Polymers onto Carbon Black Surface by Radical Trapping

The grafting of PNIPAM (or PDEAA) onto carbon black surface was achieved through the trapping of PNIPAM (or PDEAA) growing radicals, which were, formed during the polymerization initiated by AIBN, by the surface. The grafting of PNIPAM and PDEAA onto carbon black surface was determined to be $5.8 \%$ and $4.4 \%$, respectively.

These polymer-grafted carbon blacks gave a stable dispersion in various solvents of grafted polymer and readily and uniformly dispersed in various polymer matrices.

Response of Electric Resistance of Composite from PNIPAM and PDEAA-grafted Carbon Black to Contaminations in $n$-Hexane

Figure 2 shows the response of the electric resistance of the composite from PNIPAM-grafted carbon black to dioctyl phthalate in $n$-hexane solution. The electric resistance of the composite scarcely changed. On the contrary, the electric resistance of the composite suddenly increased to $10^{5}$ times of the initial resistance when the composite was dipped into $10 \mathrm{vol} \%$ dioctyl phtalate in $n$-hexane, and returned immediately to the initial resistance when it was transferred into dry air. The resistance change of the composite is reversible, and the response of electric resistance is excellently reproducible in every cycle of dipping to

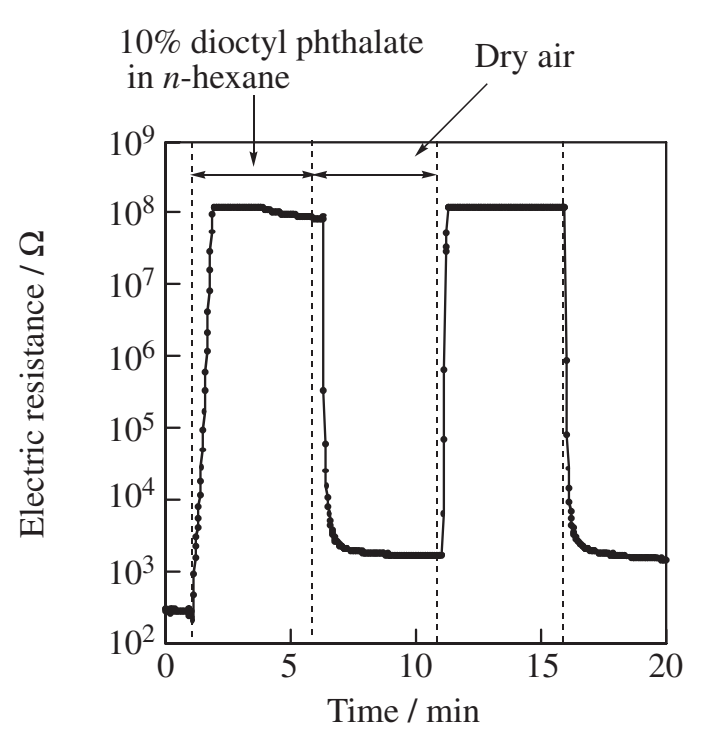

Figure 2. Response of electric resistance of the composite from PNIPAM-grafted carbon black to $10 \mathrm{vol} \%$ dioctyl phtalate in $n$-hexane solution at $25^{\circ} \mathrm{C}$. The content of carbon black in the composite is $20 \mathrm{wt} \%$.

$n$-hexane containing dioctyl phtalate and dry air.

Figure 3 shows the response of the electric resistance of the composite from PDEAA-grafted carbon black to chloroform in $n$-hexane solution. The electric resistance of the composite also drastically increased to $10^{5}$ times of the initial resistance when the composite was dipped into $10 \mathrm{vol} \%$ chloroform in $n$-hexane, and returned immediately to the initial resistance when it was transferred into dry air.

Table I shows the response of conductive composite from PNIPAM and PDEAA-grafted carbon black to various contaminations in $n$-hexane. In this study, the response in a solution was also analyzed by calculating $R / R_{0}$, where $R$ was the steady-state response value in 


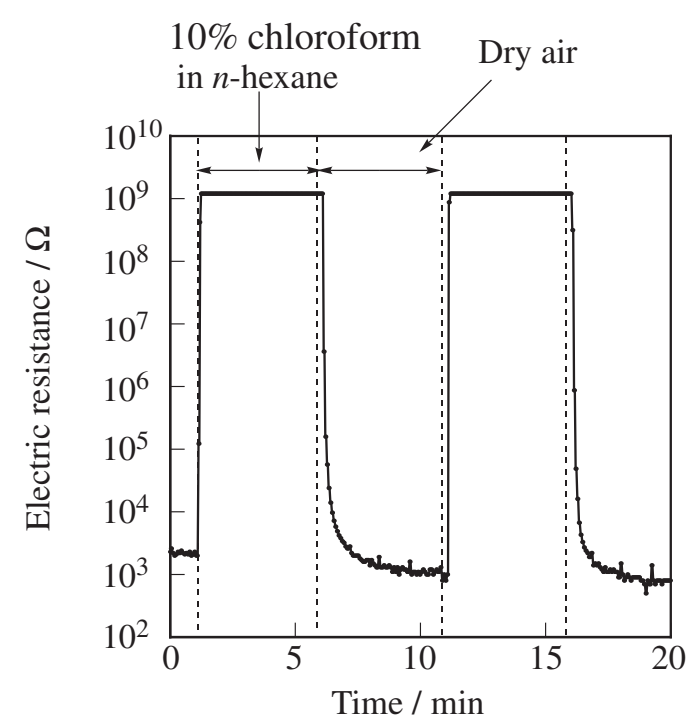

Figure 3. Response of electric resistance of the composite from PDEAA-grafted carbon black to $10 \mathrm{vol} \%$ chloroform in $n$-hexane solution at $25^{\circ} \mathrm{C}$. The content of carbon black in the composite is $20 \mathrm{wt} \%$.

Table I. Response of electric resistance of the composite from amphiphilic polymer-grafted carbon black to contaminations in $n$-hexane at $25^{\circ} \mathrm{C}$

\begin{tabular}{lcc}
\hline \multirow{2}{*}{ Contamination } & \multicolumn{2}{c}{ Response $\left(R / R_{0}\right)$} \\
\cline { 2 - 3 } Methanol & PNIPAM & PDEAA \\
$m$-Cresol & 15000 & 4000 \\
Trichloroethane & $850000^{*}$ & $19000^{*}$ \\
THF & 2.7 & $>390000$ \\
Dioctyl phthalate & 10000 & 9800 \\
Chloroform & $>410000$ & 1.1 \\
Styrene & 1.2 & $>5800000^{*}$ \\
Benzene & 1.0 & 4500 \\
Cyclohexane & 1.3 & 1.2 \\
Bisphenol A & 0.6 & 0.6 \\
Pentachlorophenol & 0.8 & 1.0 \\
\hline
\end{tabular}

*Not return to initial resistance

solution and $R_{0}$ is the baseline value of the resistance (initial resistance), taken as the resistance value just before the dip began, and the $R / R_{0}$ was expressed as the response of the electric resistance of the composite.

As shown in Table I, it was found that the response of the composite from PNIPAM-grafted carbon black was considerably high to methanol, $m$-cresol, THF, dioctyl phthalate, and chloroform in $n$-hexane solution. In the case of $m$-cresol, the electric resistance drastically increased when it was dipped into $m$-cresol in $n$-hexane solution, but did not return to initial resistance, when it was transferred in the dry air. It is considered that the $m$-cresol absorbed in PNIPAM does not dissolve out, even if it is returned in the dry air.
On the other hand, the response to trichloroethane, styrene, benzene, cyclohexane, bisphenol A, and pentachlorophenol in $n$-hexane solution is small.

The response of the composite from PDEAA-grafted carbon black was considerably high to methanol, $m$-cresol, trichloroethane, THF, chloroform, styrene and benzene in $n$-hexane solution. In the case of $m$-cresol and styrene, the electric resistance drastically increased when it was dipped into $n$-hexane solutions, but did not return to initial resistance, when it was transferred in the dry air. On the other hand, the response to dioctyl phthalate, cyclohexane, bisphenol $\mathrm{A}$, and pentachlorophenol in $n$-hexane solution is small.

Based on the above results, it was found that the response was excellent to $n$-hexane solution containing contaminations, which had high dielectric constant or were good solvents for PNIPAM and PDEAA. No correlation between sensitivity of polymers and solubility parameter of solvents was observed.

On the contrary, response of composite from PDEAA-grafted carbon black to benzene and styrene, which have low dielectric constant, was higher than that from PNIPAM-grafted carbon black.

It is considered that the solubility of hydrophobic solute in PDEAA was higher than that in PNIPAM, because PDEAA dose not have amide hydrogen bond but have ethyl group.

From the above results, various contaminations in $n$-hexane solution were able to be sensing by using the composite from PNIPAM and PDEAA-grafted carbon black.

Relationship between the Electric Resistance of the Composite from PNIPAM-grafted Carbon Black and Concentration of Contamination in n-Hexane Solution

The relationship between electric resistance of the composite from PNIPAM-grafted carbon black and chloroform concentration in $n$-hexane solution was investigated. The result was shown in Figure 4. It was found that the electric resistance increased with increasing the chloroform concentration in $n$-hexane solution. The logarithm of electric resistance is linearly proportional to chloroform concentration in $n$-hexane solution. This suggests that the composite can be used as a sensor and densitometer of chloroform in $n$-hexane.

Response of Electric Resistance of the Composite from PNIPAM and PDEAA-grafted Carbon Black to Various Solvent Vapors

Figure 5 shows the response of the electric resistance of the composite prepared from PNIPAM-grafted carbon black to methanol vapor. The electric resistance of the composite suddenly increased in methanol 


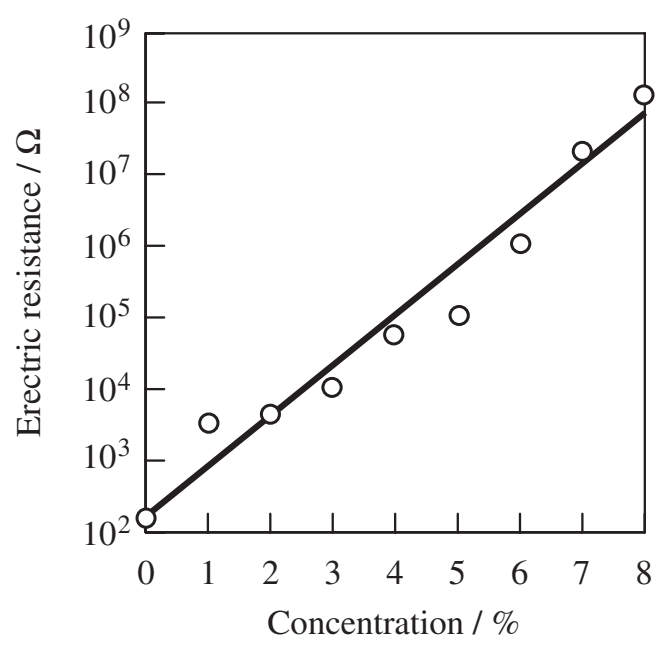

Figure 4. Relationship between the response of electric resistance of the composite from PNIPAM-grafted carbon black and the chloroform concentration in $n$-hexane solution at $25^{\circ} \mathrm{C}$. The content of carbon black in the composite is $20 \mathrm{wt} \%$.

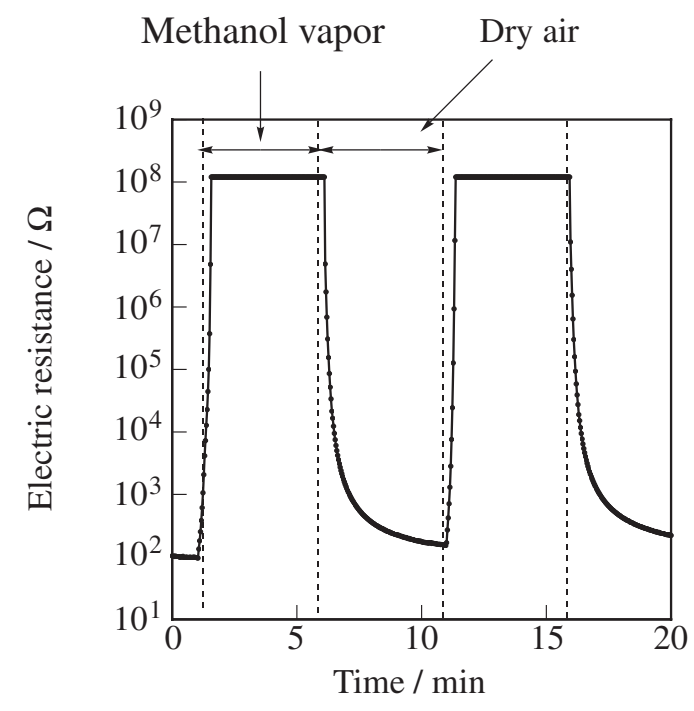

Figure 5. Response of electric resistance of the composite from PNIPAM-grafted carbon black to methanol vapor at $25^{\circ} \mathrm{C}$. The content of carbon black in the composite is $20 \mathrm{wt} \%$.

vapor, and returned immediately to the initial resistance when it was transferred into dry air.

The response of electric resistance of the composites from PNIPAM-grafted and PDEAA-grafted carbon black to various vapors was summarized in Table II. As shown in Table II, it was found that the response of the composite from PNIPAM-grafted carbon black was very large to methanol, THF, and chloroform vapor at $25^{\circ} \mathrm{C}$ under standard atmospheric pressure. On the other hand, the response to $m$-cresol, dioctyl phthalate, styrene benzene, cyclohexane, and $n$-hexane vapor was small. The response of the composite from PDEAA-grafted carbon black was also
Table II. Response of electric resistance of the composite from amphiphilic polymer-grafted carbon black to various solvent vapors at $25^{\circ} \mathrm{C}$

\begin{tabular}{lcc}
\hline \multirow{2}{*}{ Solvent } & \multicolumn{2}{c}{ Response $\left(R / R_{0}\right)$} \\
\cline { 2 - 3 } Methanol & PNIPAM & PDEAA \\
$m$-Cresol & 1150000 & 273 \\
Trichloroethane & 1.0 & 1.1 \\
THF & $>430000$ & 6.5 \\
Dioctyl phthalate & 1.1 & $>21000^{*}$ \\
Chloroform & $>960000$ & 1.3 \\
Styrene & 1.2 & $3200^{*}$ \\
Benzene & 2.0 & $>21000^{*}$ \\
Cyclohexane & 1.0 & 1.3 \\
$n$-Hexane & 1.0 & 1.1 \\
\hline
\end{tabular}

${ }^{*}$ Not return to initial resistance

very large against THF, chloroform, styrene, and benzene vapor. The drastic increase of electric resistance in these solvent vapor was observed, but the electric resistance hardly returned to initial resistance even if it was transferred to the dry air. This may be due to the high solubility of organic solvent in PDEAA. On the other hand, the response against $m$-cresol, dioctyl phthalate, cyclohexane, and $n$-hexane vapor was small.

Based on the above results, it seems that the response was excellent to solvent vapor, which had low boiling point or were good solvents for PNIPAM and PDEAA. On the contrary, response of composite from PDEAA-grafted carbon black was higher than composite from PNIPAM-grafted carbon black against benzene and styrene, which had high boiling point. No correlation between sensitivity of polymers and solubility parameter of solvents was observed.

It is considered that the solubility of hydrophobic solute in PDEAA was higher than that in PNIPAM, because PDEAA dose not have amide hydrogen bond and but have ethyl group.

Relationship between Electric Resistance of the Composite from PNIPAM-grafted Carbon Black and Relative Humidity

The effect of relative humidity on the electric resistance of the composite from PNIPAM-grafted carbon black was investigated. The result was shown in Figure 6. It was found that the electric resistance increased with increasing the humidity. In addition, the logarithm of responsiveness is linearly proportional to humidity. It was found that the composite from PNIPAM-grafted carbon black with PNIPAM could measure humidity from about 0 to $100 \%$. This suggests that this composite can be used as a sensor and hygrometer. 


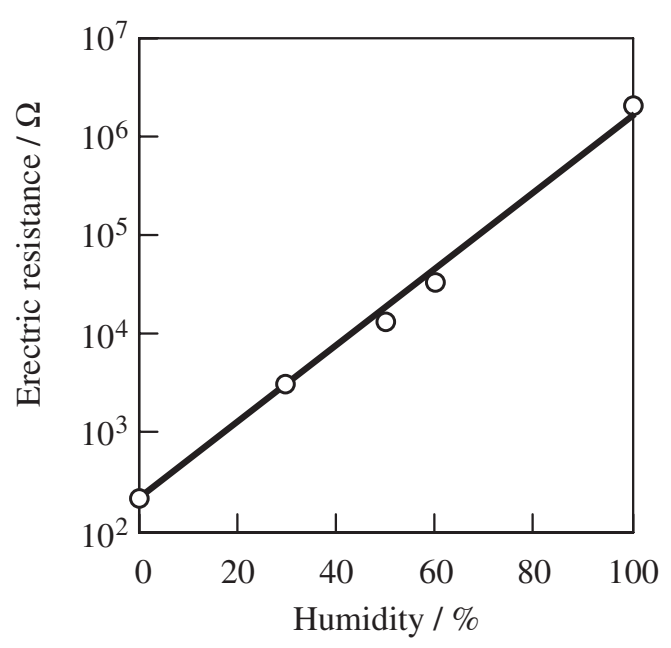

Figure 6. Relationship between electric resistance of the composite from PNIPAM-grafted carbon black and relative humidity at $25^{\circ} \mathrm{C}$. The content of carbon black in the composite is $20 \mathrm{wt} \%$.

\section{CONCLUSIONS}

1. The electric resistance of the composites from PNIPAM-grafted carbon black and PDEAAgrafted carbon black drastically increased, when it was dipped in $n$-hexane containing contaminations, such as methanol, THF, and chloroform and returned immediately to the initial resistance when it was transferred into dry air.

2. The electric resistance of the composites from PNIPAM-grafted carbon black drastically increased, when it was exposed to the solvent vapor of methanol, THF, and chloroform at $25^{\circ} \mathrm{C}$, and immediately returned to initial resistance when it was transferred to dry air.

3. The response of electric resistance of the composite from PNIPAM-grafted carbon black was found to be linearly proportional to chloroform as contamination in $n$-hexane solution and relative humidity.
Acknowledgment. This study was partly supported by a Grant in Aid for Scientific Research from the Ministry of Education, Culture, Sports, Science and Technology of Japan (No. 15360357), which is gratefully acknowledged.

\section{REFERENCES}

1. N. Tsubokawa, Prog. Polym. Sci., 17, 417 (1992).

2. N. Tsubokawa, in "Fundamental and Applied Aspects of Chemically Modified Surfaces": J. P. Bilz and C. B. Little, Ed., The Royal Society of Chemistry, Cambridge, pp. 36, 1999.

3. N. Tsubokawa, Bull. Chem. Soc. Jpn., 75, 2115 (2002).

4. S. Hayashi, A. Naitoh, S. Machida, M. Okazaki, K. Maruyama, and N. Tsubokawa, Appl. Organomet. Chem., 12, 743 (1998).

5. N. Tsubokawa, Y. Shirai, M. Okazaki, and K. Maruyama, Polym. Bull., 42, 425 (1999).

6. M. Okazaki, K. Maruyama, M. Tsuchida, and N. Tsubokawa, Polym. J., 31, 672 (1999).

7. J. Chen and N. Tsubokawa, Polym. Adv. Technol., 11, 101 (2000).

8. J. Chen and N. Tsubokawa, Polym. J., 32, 729 (2000).

9. M. Narkis, S. Srivastava, R. Tchoudakov, and O. Breuer, Synth. Met., 113, 29 (2000).

10. B. J. Doleman, M. C. Lonergan, E. J. Severin, T. P. Vaid, and N. S. Lewis, Anal. Chem., 70, 4177 (1998).

11. E. J. Severin, B. J. Doleman, and N. S. Lewis, Anal. Chem., 72, 658 (2000).

12. X. M. Dong, R. W. Fu, M. Q. Zhang, B. Zhang, J. R. Li, and M. Z. Rong, Carbon, 41, 371 (2003).

13. X. M. Dong, R. W. Fu, M. Q. Zhang, B. Zhang, J. R. Li, and M. Z. Rong, Polym. Bull., 50, 99 (2003).

14. J. R. Li, J. R. Xu, M. Q. Zhang, and M. Z. Rong, Carbon, 41, 2353 (2003).

15. X. M. Dong, R. W. Fu, M. Q. Zhang, B. Zhang, J. R. Li, and M. Z. Rong, J. Mater. Sci. Lett., 22, 1057 (2003).

16. N. Tsubokawa, M. Tsuchida, J. Chen, and Y. Nakazawa, Sens. Actuators, B, 79, 92 (2001). 